\title{
Cutaneous Chaetomium globosum Infection in a Vedolizumab-Treated Patient
}

\author{
Morgan Cronin, MD; Timothy G. Berger, MD; Stuart H. Cohen, MD; Uma Mahadevan, MD; \\ Jeffery P. North, MD; Sarah Asch, MD
}

\section{PRACTICE POINTS}

- Tissue culture remains the gold standard for deep fungal infections.

- Physicians must maintain a high index of suspicion for alternate diagnoses when a disease progresses along an unexpected course.

- Biologic medications may have low-incidence side effects that emerge in postmarket use.

To the Editor:

Broader availability and utilization of novel biologic treatments has heralded the emergence of unusual infections, including skin and soft tissue infections. These unusual infections may not be seen in clinical trials due to their overall rare incidence. In modern society, exposure to unusual pathogens can occur in locations far from their natural habitat. ${ }^{1}$ Tissue culture remains the gold standard, as histopathology and smears may not identify the organisms. Tissue culture of these less-common pathogens is challenging and may require multiple samples and specialized laboratory evaluations. ${ }^{2}$ In some cases, a skin biopsy with histopathologic examination is an efficient means to confirm or exclude a dermatologic manifestation of an inflammatory disease. This information can quickly change the course of treatment, especially for those on immunosuppressive medications. ${ }^{3}$ We report a case of unusual cutaneous infection with Chaetomium globosum in a patient concomitantly treated with vedolizumab, a gut-specific integrin inhibitor, alongside traditional immunosuppressive therapy.

A 33-year-old woman with Crohn disease on vedolizumab and mercaptopurine was referred to the dermatology clinic with firm, tender, erythematous lesions on the legs of 1 month's duration (Figure, A). She had a history of inflammatory bowel disease with perianal fistula, sacroiliitis, uveitis, guttate psoriasis, and erythema nodosum. She denied recent medication changes, foreign travel, swimming in freshwater or a hot tub, chills, fever, malaise, night sweats, and weight loss. Physical examination revealed several tender, indurated, erythematous plaques across the legs, ranging in size from 4 to $12 \mathrm{~cm}$. The plaques had central hyperpigmentation, atrophy, and scant scale without ulceration, drainage, or pustules. The largest plaque demonstrated a well-defined area of central fluctuance. Prednisone $(60 \mathrm{mg})$ with taper was initiated for presumed recurrence of erythema nodosum with close follow-up.

Five weeks later, most indurated plaques healed, leaving depressed scars; however, at $10 \mathrm{mg}$ of prednisone she developed 2 additional nodules on the shin that, unlike earlier plaques, developed a central pustule and drained. The prednisone dose was increased to control the new areas and tapered thereafter to $20 \mathrm{mg}$ daily. Despite the

Dr. Cronin is from the Department of Pediatrics; Dr. Berger is from the Department of Dermatology; Dr. Mahadevan is from the Department of Medicine, Division of Gastroenterology; and Dr. North is from Department Dermatology, all at the University of California, San Francisco. Dr. Cohen is from Division of Infectious Disease, University of California, Davis. Dr. Asch is from the Department of Dermatology, HealthPartners and Park Nicollet Medical Groups, St. Paul, Minnesota.

Drs. Cronin, Berger, Cohen, North, and Asch report no conflict of interest. Dr. Mahadevan is a consultant for Takeda Pharmaceutical Company. Correspondence: Sarah Asch, MD, 401 Phalen Blvd, St. Paul, MN 55130 (sarah@asch.org).

doi:10.12788/cutis.0329 

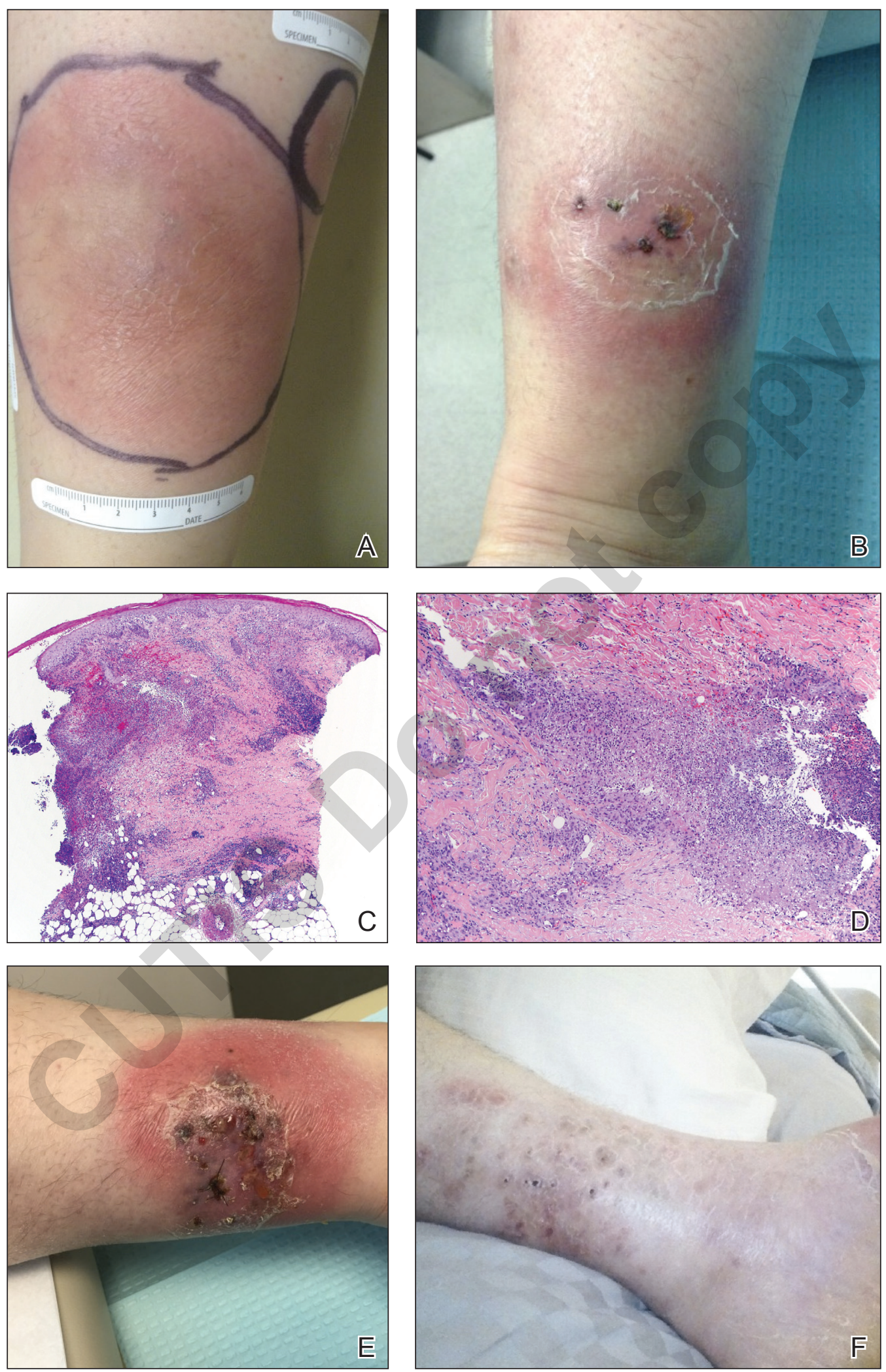

A, Chaetomium globosum infection at presentation. B, The patient experienced increased edema, and a tissue culture was taken 8 weeks after presentation. C and D, Histopathology revealed suppurative and granulomatous dermatitis (H\&E, original magnifications $\times 40$ and $\times 100$ ). E, Itraconazole was started, and unspecified mold was found on tissue culture 10 weeks after presentation. F, Slow improvement was seen 14 weeks after presentation. 
overall improvement, 2 plaques remained on the left side of the shin. Initially, erythema nodosum recurrence was considered, given the setting of inflammatory bowel disease and recent more classic presentation ${ }^{4}$; however, the disease progression and lack of response to standard treatment suggested an alternate pathology. Further history revealed that the patient had a pedicure 3 weeks prior to initial symptom onset. A swab was sent for routine bacterial culture at an outside clinic; no infectious agents were identified.

Three weeks later, the patient's condition had worsened again with increased edema, pain with standing, and more drainage (Figure, B). She did not report fevers or joint swelling. A punch biopsy was performed for tissue culture and histopathologic evaluation, which revealed granulomatous and suppurative inflammation and excluded erythema nodosum. Special stains for organisms were negative (Figure, C and D). Two weeks later, tissue culture began growing an unspecified mold. Mercaptopurine and prednisone were immediately discontinued. The patient remained on vedolizumab, started itraconazole $(200 \mathrm{mg})$, and was referred to an infectious disease (ID) specialist. The sample was eventually identified as $C$ globosum (Figure, E) at a specialized facility (University of Texas, San Antonio). Despite several weeks of itraconazole therapy, the patient developed edema surrounding the knee. Upon evaluation by orthopedics, the patient was diagnosed with reactive arthritis in the left knee and ankle. The knee fluid was drained, and cultures were negative. At recommendation of the ID physician, the itraconazole dosage was doubled given the limited clinical response. After several weeks at the increased dosage, she began to experience slow improvement (Figure, F). Because Chaetomium species infections are rare and have limited response to many antifungal agents, ${ }^{5}$ no standard treatment protocol was available. Initial recommendations for treatment were for 1 year, based on the experience and expertise of the ID physician. Treatment with itraconazole was continued for 10 months, at which point the patient chose to discontinue therapy prior to her follow-up appointments. The patient had no evidence of infection recurrence 2 months after discontinuing therapy.

In the expanding landscape of targeted biologic therapies for chronic inflammatory disease, physicians of various specialties are increasingly encountering unanticipated cutaneous eruptions and infections. Chaetomium is a dematiaceous mold found primarily in soil, water, decaying plants, paper, or dung. Based on its habitat, populations at risk for infection with Chaetomium species include farmers (plant and animal husbandry), children who play on the ground, and people with inadequate foot protection. ${ }^{1,2}$ Chaetomium globosum has been identified in indoor environments, such as moldy rugs and mattresses. In one report, it was cultured from the environmental air in a bone marrow transplant patient's room after the patient presented with delayed infection. ${ }^{6}$
Although human infection is uncommon, clinical isolation of Chaetomium species has occurred mainly in superficial samples from the skin, hair, nails, eyes, and respiratory tract. ${ }^{1}$ It been reported as a causative agent of onychomycosis in several immunocompetent patients ${ }^{7,8}$ but rarely is a cause of deep-skin infection. Chaetomium is thought to cause superficial infections, as it uses extracellular keratinases ${ }^{1}$ to degrade protective keratin structures, such as human nails. Infections in the brain, blood, and lymph nodes also have been noted but are quite rare. Deep skin infections present as painful papules and nodules to nonhealing ulcers that develop into inflammatory granulomas on the extremities. ${ }^{3}$ Local edema and yellow-brown crust often is present and fevers have been reported. Hyphae may be identified in skin biopsy. ${ }^{8}$ We posit that our patient may have been exposed to Chaetomium during her pedicure, as recirculating baths in nail salons have been a reported site of other infectious organisms, such as atypical mycobacteria. ${ }^{9}$

Vedolizumab is a humanized IgG1 monoclonal antibody used in the treatment of ulcerative colitis and Crohn disease. It targets the $\alpha 4 \beta 7$ integrin, a specific modulator of gut-trafficking lymphocytes. In vedolizumab's clinical trial for Crohn disease, there was no increased incidence of life-threatening, severe infection. ${ }^{10,11}$ Often, new biologic treatments are used with known immunosuppressive medications. Mercaptopurine and prednisone are implicated in infections; however, recovery from the immune suppression usually is seen at 1 month after discontinuation. ${ }^{12}$ Our patient continued to worsen for several weeks and required increased dosing of itraconazole, despite stopping both prednisone and mercaptopurine. It opens the question as to whether vedolizumab played a role in the recalcitrant disease.

This case illustrates the importance of a high index of suspicion for unusual infections in the setting of biologic therapy. An infectious etiology of a cutaneous eruption in an immunosuppressed patient should always be included in the differential diagnosis and actively pursued early on; tissue culture may shorten the treatment course and decrease severity of the disease. Although a direct link between the mechanism of action of vedolizumab and cutaneous infection is not clear, given the rare incidence of this infection, a report of such a case is important to the practicing clinician.

\section{REFERENCES}

1. de Hoog GS, Ahmed SA, Najafzadeh MJ, et al. Phylogenetic findings suggest possible new habitat and routes of infection of human eumycetoma. PLoS Negl Trop Dis. 2013;7:e2229. doi:10.1371/journal .pntd.0002229

2. Zhang H, Ran Y, Li D, et al. Clavispora lusitaniae and Chaetomium atrobrunneum as rare agents of cutaneous infection. Mycopathologia. 2010;169:373-380. doi:10.1007/s11046-009-9266-9

3. Schieffelin JS, Garcia-Diaz JB, Loss GE, et al. Phaeohyphomycosis fungal infections in solid organ transplant recipients: clinical presentation, pathology, and treatment. Transpl Infect Dis Off J Transplant Soc. 2014;16:270-278. doi:10.1111/tid.12197

4. Farhi D, Cosnes J, Zizi N, et al. Significance of erythema nodosum and pyoderma gangrenosum in inflammatory bowel 
diseases: a cohort study of 2402 patients. Medicine (Baltimore). 2008;87:281-293. doi:10.1097/MD.0b013e318187cc9c

5. Guarro J, Soler L, Rinaldi MG. Pathogenicity and antifungal susceptibility of Chaetomium species. Eur J Clin Microbiol Infect Dis Off Publ Eur Soc Clin Microbiol. 1995;14:613-618.

6. Teixeira ABA, Trabasso P, Moretti-Branchini ML, et al. Phaeohyphomycosis caused by Chaetomium globosum in an allogeneic bone marrow transplant recipient. Mycopathologia. 2003;156:309-312.

7. Falcón CS, Falcón MDMS, Ceballos JD, et al. Onychomycosis by Chaetomium spp. Mycoses. 2009;52:77-79. doi:10.1111 /j.14390507.2008.01519.x

8. Kim DM, Lee MH, Suh MK, et al. Onychomycosis caused by Chaetomium globosum. Ann Dermatol. 2013;25:232-236. doi:10.5021/ ad.2013.25.2.232
9. Vugia DJ, Jang Y, Zizek C, et al. Mycobacteria in nail salon whirlpool footbaths, California. Emerg Infect Dis. 2005;11:616-618. doi:10.3201 leid1104.040936

10. Luthra P, Peyrin-Biroulet L, Ford AC. Systematic review and meta-analysis: opportunistic infections and malignancies during treatment with anti-integrin antibodies in inflammatory bowel disease. Aliment Pharmacol Ther. 2015;41:1227-1236. doi:10.1111 lapt.13215

11. Colombel J-F, Sands BE, Rutgeerts P, et al. The safety of vedolizumab for ulcerative colitis and Crohn's disease. Gut. 2017;66:839-851. doi:10.1136/gutjnl-2015-311079

12. Connell WR, Kamm MA, Ritchie JK, et al. Bone marrow toxicity caused by azathioprine in inflammatory bowel disease: 27 years of experience. Gut. 1993;34:1081-1085. 